\title{
SPH SIMULATIONS OF TIDALLY UNSTABLE ACCRETION DISKS
}

\author{
JAMES MURRAY \\ Canadian Institute for Theoretical Astrophysics \\ University of Toronto \\ Ontario M5S 1A7, Canada \\ jmurray@cita.utoronto.ca
}

We use two dimensional Smoothed Particle Hydrodynamics simulations to investigate the the Tidal Resonance Instability (TRI) model for superhumps in SU UMa stars.

In the TRI model, superhumps are the signature of an eccentric disk being periodically stressed by the binary system's rotating tidal field. A slow prograde motion of the disk in the inertial frame is then responsible for the superhump period slightly exceeding the binary orbital period. The disk eccentricity is caused by a 3:1 resonance between orbits in the disk and the orbit of the binary itself. The development of the TRI model is due mainly to Whitehurst (1988), Hirose \& Osaki (1990) and Lubow (1991a). Lubow in particular carried out a nonlinear mode analysis of a fluid disk's response to a weak tidal field and identified the feedback mechanism responsible for disk eccentricity growth.

The tidal field due to the secondary star can be decomposed into a set of functions $\phi_{m}(r) \cos \left[m\left(\theta-\Omega_{\mathrm{b}} t\right)\right]$, where $m$ (an integer) is the mode number, and $\Omega_{\mathrm{b}}$ is the angular speed of the secondary about the primary. The $m$ th component of the tidal field generates a sinusoidal response with argument $m\left(\theta-\Omega_{\mathrm{b}} t\right)$. At the 3:1 eccentric inner Lindblad resonance the disk's $m=3$ tidal response couples with any existing eccentricity in the disk to produce a travelling wave (argument $2 \theta-3 \Omega_{\mathrm{b}} t$ ). In turn, the interaction of the travelling wave with the tidal response reinforces the disk eccentricity. Lubow (1991a) calculated the initial exponential rate of growth of the eccentricity.

SPH simulations performed to test the analysis (Lubow 1991b, 1992) showed that the disk's tidal response to the $m=2$ component of the binary potential interfered with the eccentricity growth mechanism by clearing material out of the Lindblad resonance. Lubow observed a much reduced eccentricity growth rate in simulations that included the $m=2$ term.

A. Evans and J. H. Wood (eds.), Cataclysmic Variables and Related Objects, 115-116.

(1) 1996 Kluwer Academic Publishers. Printed in the Netherlands. 
In this poster, the results of two SPH accretion disk simulations are described. System parameters $\left(q=\frac{3}{17}, \dot{M}=10^{-9} \mathrm{M}_{\odot} \mathrm{yr}^{-1}, T=2 \pi \Omega_{\mathrm{b}}^{-1}=\right.$ $0.063121 \mathrm{~d}$ ) were chosen to be appropriate for OY Car in (super)outburst. For an introduction to SPH the reader is referred to Monaghan (1992). For details of these simulations and tests of the code see Murray (1996).

In the first calculation, a disk was built up via a steady mass transfer stream originating at the $L_{1}$ point. In the second simulation, mass was added to the disk in Keplerian orbit at the disk circularisation radius $r_{c}$. Both simulations were isothermal with sound speed $c=0.02 d \Omega_{\mathrm{b}}$, where $d$ is the inter-star separation. The kinematic viscosity $\nu=2.510^{-4} d^{2} \Omega_{\mathrm{b}}$. The calculations were followed for over 160 binary periods. In that time the disks reached an eccentric, precessing equilibrium state. Mode strengths in the disk were determined using a Fourier analysis similar to that in Lubow (1991b).

For this particular system, theory predicts an eccentricity growth rate $\lambda / \Omega_{\mathrm{b}}=0.10 \pm 0.01$. In both simulations, the growth of the eccentricity was well fit by an exponential curve. With the $L_{1}$ stream the growth rate was $\lambda_{1} / \Omega_{\mathrm{b}}=0.046 \pm 0.002$. In the second simulation $\lambda_{2} / \Omega_{\mathrm{b}}=0.058 \pm 0.002$. These values are approximately half the predicted value. Adding mass to the disk from $L_{1}$ further reduced the rate. However our simulations did not show the much diminished eccentricity growth described in Lubow (1992). In fact we observed that as our disks became more eccentric, the tidal response to the $m=2$ component of the binary potential became weaker.

The eccentricity grew sufficiently rapidly to agree with superhump observations. In particular, luminosity variations associated with the disk precession developed in both calculations within a day or so of the disk encountering the Lindblad resonance. Superhumps appear approximately a day into superoutburst.

\section{References}

Hirose, M., Osaki, Y., 1990, PASJ, 42, 135

Lubow, S.H., 1991a, Ap. J., 381, 259

Lubow, S.H., 1991b, Ap. J., 381, 268

Lubow, S.H., 1992, Ap. J., 401, 317

Monaghan, J.J., 1992, ARAA, 30, 543

Murray, J.R., 1996, MNRAS, in press

Whitehurst, R., 1988, MNRAS, 232, 35 